$\mathrm{UMH}-\mathrm{MG}-00 / 02$

November 14, 2018

\title{
About maximally localized states in quantum mechanics
}

\author{
S. Detournay, C. Gabriel[.|[ and Ph. Spindel] \\ Mécanique et Gravitation \\ Université de Mons-Hainaut, 20 Place du Parc \\ 7000 Mons, Belgium
}

\begin{abstract}
We analyze the emergence of a minimal length for a large class of generalized commutation relations, preserving commutation of the position operators and translation invariance as well as rotation invariance (in dimension higher than one). We show that the construction of the maximally localized states based on squeezed states generally fails. Rather, one must resort to a constrained variational principle.
\end{abstract}

Keywords: maximally localized states, squeezed state, variational principle PACS: 03.65.Ca,03.65.Db

\section{Introduction}

In a series of articles ([1]-[3]), Kempf and collaborators have studied the quantum mechanical implications of a modification of the usual canonical commutation relations which is inspired by noncommutative geometry [3] and string theory (see for instance refs 柾). Assuming that $[\hat{\mathbf{x}}, \hat{\mathbf{p}}]=i f(\hat{\mathbf{x}}, \hat{\mathbf{p}})$ these authors show that a minimal length appears naturally from the Heisenberg uncertainty relation

$$
\Delta \hat{\mathbf{x}} \Delta \hat{\mathbf{p}} \geq \frac{1}{2}|\langle[\hat{\mathbf{x}}, \hat{\mathbf{p}}]\rangle| .
$$

This crucial modification of fundamental commutators is physically grounded, and lies at the basis of recent theoretical models aiming at describing quantum geometry or quantum gravity. The emergence of a minimal localization length seems indeed to be a recurrent feature when trying to approach quantum gravity and has far reaching consequences (see e.g. [0] and references therein). This short distance structure could for instance allow to get rid of ultraviolet divergencies (see [6] for a discussion in the context of modified commutation relations). The introduction of a naturel ultraviolet cutoff could also affect the spectrum of the cosmological microwave background [7, 8]. We will focus in this

\footnotetext{
${ }^{1}$ Postdoctoral researcher of the Belgium National Fund for Scientific Research.

${ }^{2}$ E-mail : claude.gabriel@umh.ac.be

3 E-mail : philippe.spindel@umh.ac.be
} 
note on the physical states displaying the minimal length uncertainty, the socalled maximally localized states. In reference [2], Kempf, Mangano and Mann (K. M. M. hereafter) claim to obtain such states as particular squeezed states. We shall show that it is only in a few special cases that maximally localized states can be obtained as squeezed states but that, in general, it is a variational principle that leads to more satisfactory results. However we wish to emphasize that our work only aims to provide what we believe to be a better definition of maximally localized states, but the credit for the idea that a modification of the canonical commutation relation leads to a fundamental minimal length belongs to the authors of refs [1]-[3].

Our paper is organized as follows. We restrict ourselves to space translation invariant situation i.e. we assume the commutator to depend only on $\hat{\mathbf{p}}$. In section $\mathbf{2}$ we analyze the K. M. M. construction and display its weaknesses. Section 3 consists in a discussion of a definition of physical maximally localized states, based on a minimization principle of the spread in space of the states (in one dimension). Section 4 illustrates some physical aspects of our analysis through study of the quantization of the particle in a box as well as the asymptotic energy eigenvalues of the harmonic oscillator, independently of the details of the commutation relation. In section $\mathbf{5}$ we show that the extension to translation invariant modified commutation relations in an arbitrary number of dimensions, under the assumption of commuting position operators, can be completely described by the use of a single function. Moreover assuming rotational invariance, we then compare the maximally localized states defined by our variational principle to those proposed by K. M. M. and show that ours lead to a finer spatial localization. We conclude our work in section 6 by a brief summary of our results.

\section{K. M. M. construction}

Hereafter we assume the operator function $f$ to be only function of $\hat{\mathbf{p}}$; this insures the space translation invariance of the commutation relation. In $p$ representation, the function $f(p)$ has to be real. Moreover we assume it to be everywhere greater or equal to a strictly positive constant

$$
\exists C>0, \forall p \in \mathbb{R}: f(p)>C
$$

i.e. whatever is the state, the operators $\hat{\mathbf{x}}$ and $\hat{\mathbf{p}}$ acting on it, never commute. In a first step let us restrict ourselves to the one dimensional problem

$$
[\hat{\mathbf{x}}, \hat{\mathbf{p}}]=i f(\hat{\mathbf{p}})
$$

In a $p$-representation these operators are realized as

$$
\hat{\mathbf{x}}=i f(p) \partial_{p} \quad \text { and } \quad \hat{\mathbf{p}}=p \in \mathbb{R} \quad .
$$

K. M. M. construction of maximally localized state consists in taking among the squeezed states, i.e. states that satisfy the relation

$$
\Delta \hat{\mathbf{x}} \Delta \hat{\mathbf{p}}=\frac{1}{2}|\langle[\hat{\mathbf{x}}, \hat{\mathbf{p}}]\rangle| \quad,
$$


the one leading to the minimal value of $\Delta \hat{\mathbf{x}}$. Squeezed states depend on three parameters, denoted $\xi, \eta$ and $\Lambda$, and are the eigenstates $\left|\Psi_{\Lambda}\right\rangle$, with vanishing eigenvalue, of the operators $\hat{\mathbf{A}}_{\Lambda}$ defined as

$$
\hat{\mathbf{A}}_{\Lambda}\left|\Psi_{\Lambda}\right\rangle \equiv[(\hat{\mathbf{x}}-\xi)+i \Lambda(\hat{\mathbf{p}}-\eta))\left|\Psi_{\Lambda}\right\rangle=0
$$

i.e. in $p$-representation

$$
\left\langle p \mid \Psi_{\Lambda}\right\rangle \propto \exp [(\Lambda \eta-i \xi) z(p)-\Lambda u(p)]
$$

where

$$
z(p)=\int_{0}^{p} f^{-1}(q) d q \quad \text { and } \quad u(p)=\int_{0}^{p} q f^{-1}(q) d q \quad .
$$

These states are always normalizable when the values of $z( \pm \infty)$ are finite, otherwise $\Lambda$ must be positive, and it is easy to check that the parameters $\xi$ and $\eta$ correspond to the expectation values of the operators $\hat{\mathbf{x}}$ and $\hat{\mathbf{p}}$ if and only if

$$
\Lambda>0 \quad \text { and } \quad \lim _{p \rightarrow \pm \infty} u(p)=\infty \quad .
$$

Now, let us compute the norm of the state $\hat{\mathbf{A}}_{\ell}\left|\Psi_{\Lambda}\right\rangle$. We obtain

$$
\begin{aligned}
\left\langle\Psi_{\Lambda} \hat{\mathbf{A}}_{\ell} \mid \hat{\mathbf{A}}_{\ell} \Psi_{\Lambda}\right\rangle & =(\ell-\Lambda)^{2}\left\langle\Psi_{\Lambda}\left|(\hat{\mathbf{p}}-\eta)^{2}\right| \Psi_{\Lambda}\right\rangle \\
& \geq 0
\end{aligned}
$$

and moreover, if (and only if) $u(|p|)$ grows quickly enough at infinity so that we have

$$
\lim _{p \rightarrow \pm \infty} p \exp [2 \Lambda(\eta z(p)-u(p))]=0 \quad .
$$

we also deduce, after an integration by parts, that:

$$
\begin{aligned}
\left\langle\Psi_{\Lambda} \hat{\mathbf{A}}_{\ell} \mid \hat{\mathbf{A}}_{\ell} \Psi_{\Lambda}\right\rangle & =\left\langle\Psi_{\Lambda} \mid \hat{\mathbf{A}}_{\ell}^{\dagger} \hat{\mathbf{A}}_{\ell} \Psi_{\Lambda}\right\rangle \\
& =\left\langle\Psi_{\Lambda}\left|(\hat{\mathbf{x}}-\xi)^{2}-\ell f(\hat{\mathbf{p}})+\ell^{2}(\hat{\mathbf{p}}-\eta)^{2}\right| \Psi_{\Lambda}\right\rangle
\end{aligned}
$$

Introducing $(\Delta \hat{\mathbf{x}})_{\Lambda}=\sqrt{\left\langle\Psi_{\Lambda}\left|\hat{\mathbf{x}}^{2}\right| \Psi_{\Lambda}\right\rangle /\left\langle\Psi_{\Lambda} \mid \Psi_{\Lambda}\right\rangle-\xi^{2}}$ and a similar definition for $(\Delta \hat{\mathbf{p}})_{\Lambda}^{2}$, we obtain by identifying eq.(10) with eq. (14) that:

$$
\begin{aligned}
(\Delta \hat{\mathbf{x}})_{\Lambda}^{2}(\Delta \hat{\mathbf{p}})_{\Lambda}^{2} & =\frac{1}{4}\langle f(\hat{\mathbf{p}})\rangle_{\Lambda}^{2} \\
\Lambda & =\frac{\langle f(\hat{\mathbf{p}})\rangle_{\Lambda}}{2(\Delta \hat{\mathbf{p}})_{\Lambda}^{2}} \\
(\Delta \hat{\mathbf{x}})_{\Lambda}^{2} & =\frac{1}{4} \frac{\langle f(\hat{\mathbf{p}})\rangle_{\Lambda}^{2}}{(\Delta \hat{\mathbf{p}})_{\Lambda}^{2}}=\Lambda^{2}(\Delta \hat{\mathbf{p}})_{\Lambda}^{2}
\end{aligned}
$$

Accordingly, if $\langle f(\hat{\mathbf{p}})\rangle_{\Lambda}^{2} /(\Delta \hat{\mathbf{p}})_{\Lambda}^{2}$ admits a strictly positive lower bound, we deduce the existence of a minimal uncertainty on the position operator on the subset of 
squeezed states. The evaluation a priori of this minimum is not simple except in two cases: if the mean values $\langle f(\hat{\mathbf{p}})\rangle_{\Lambda}$ are bounded, in which case the minimum is zero, or if $f(\hat{\mathbf{p}})=1+\beta^{2} \hat{\mathbf{p}}^{2}$, the case discussed in detail by K. M. M. [2]. To avoid unnecessary clutter, in the following we shall made use of units such that constants, like $\beta$ in the previous equation, are set equal to unity. When the previous assumptions $(9,12)$ are verified, in the $p$-representation we may obtain a lower bound for the space uncertainty of the squeezed states by calculating the minimum (with respect to $\Lambda$ and $\eta$ ) of the integral expression of $(\Delta \hat{\mathbf{x}})_{\Lambda}$ :

$$
(\Delta \hat{\mathbf{x}})_{\Lambda}^{2}=\Lambda^{2} \frac{\int_{-\infty}^{+\infty}\left(p^{2}-\eta^{2}\right) \exp [-2 \Lambda(u(p)-\eta z(p))] f^{-1}(p) d p}{\int_{-\infty}^{+\infty} \exp [-2 \Lambda(u(p)-\eta z(p))] f^{-1}(p) d p}
$$

In particular, under our assumption that for large values of $|p|$, the function $f(p)$ behaves as $|p|^{1+\nu}$ — with $-1 \leq \nu \leq 1$ in order to admit a strictly positive lower bound and to satisfy the conditions (9, 12) — we may check that for $\Lambda$ going to zero we obtain:

$$
(\Delta \hat{\mathbf{x}})_{\Lambda}^{2} \propto\left\{\begin{array}{l}
\Lambda^{\frac{2 \nu}{\nu-1}} \rightarrow 0 \text { if } \nu<0 \\
\Lambda^{\frac{\nu}{\nu-1}} \rightarrow \infty \text { if } 0<\nu<1
\end{array}\right.
$$

When $\nu=0$, according to the precise behavior of $f(p),(\Delta \hat{\mathbf{x}})_{\Lambda}^{2}$ will converge or diverge. If $\nu=1$, the condition (12) imposes to restrict the domain of $\Lambda$ to values greater than $1 / 2$. Moreover in the limit $\Lambda \rightarrow 1 / 2,(\Delta \hat{\mathbf{x}})_{1 / 2}^{2}$ diverges, while when $\Lambda$ goes to infinity, as a saddle point estimate shows that $(\Delta \hat{\mathbf{x}})_{\Lambda}^{2}$ grows as $\Lambda$ and therefore diverges again. As a consequence, for positive values of $\nu \leq 1$, $(\Delta \hat{\mathbf{x}})_{\Lambda}^{2}$ reaches its minimum for a finite, non-zero, value of $\Lambda$. This minimum must be strictly positive, otherwise we obtain also $(\Delta \hat{\mathbf{p}})_{\Lambda}^{2}=0$ and $\langle f(\hat{\mathbf{p}})\rangle_{\Lambda}=0$, which is of course impossible. In the special case, $f(p)=1+2 k p+p^{2}$ it is trivial to obtain the expression of the position uncertainty :

$$
(\Delta \hat{\mathbf{x}})_{\Lambda}^{2}=\frac{\Lambda^{2}}{2 \Lambda-1}\left(1+2 k \eta+\eta^{2}\right)
$$

Minimizing this expression with respect to the variables $\Lambda$ and $\eta$ yields $(\Delta \hat{\mathbf{x}})^{2}=$ $1-k^{2}>0$ for $\Lambda=1$ and $\eta=-k$.

However it is clear that the K. M. M. approach consists in the search for a possible maximally localized states only among the subset of states that saturate the Heisenberg inequality (5): the squeezed states. But when modified commutation relations are involved, it is worthwhile to remark that in contrast to what happens in the framework of usual quantum mechanic, where $f(\hat{\mathbf{p}})=\mathbb{I}$ and therefore $\langle f(\hat{\mathbf{p}})\rangle=1$ whatever is the state, the expectation value of the operator $f(\hat{\mathbf{p}})$, in general, depends on the state considered. Thus nothing prevents a minimal length smaller than the one obtained by considering only squeezed

\footnotetext{
${ }^{4}$ In principle, we have to introduce two different exponents $\nu_{-}$and $\nu_{+}$that will reflect the behaviors of $f(p)$ near $+\infty$ and $-\infty$; we shall not do it to lighten the text and just make use of one, denoted by $\nu$.
} 
states, simply because there may be physically relevant states for which $\Delta \hat{\mathbf{x}}^{2}$ takes on a lower value than its lower bound on the subset of squeezed states.

Moreover it is only for a restricted class of functions $f(p)$, those that do not grow faster than $|p|^{2}$, that squeezed states may be used in the chain of relations (13) 14). For instance, if we assume $f(p)=1+|p|^{\nu}$ with $\nu>3$, then all the integrals appearing in eqs (18) converge for all non negative values of $\Lambda$ and thus the minimal value of $(\Delta \hat{\mathbf{x}})_{\Lambda}^{2}$ so estimated is zero. Taking for instance $f(p)=1+p^{4}$, this seems to be in contradiction with eq. (75). But after a rapid glance, it is clear that there is actually no "contradiction": it just is that the mean values $\left\langle\hat{\mathbf{x}}^{2}\right\rangle_{\Lambda}$ and $\langle f(\hat{\mathbf{p}})\rangle_{\Lambda}$ do not exist. In the next section we shall reformulate the problem of the existence of a physical minimal length on a more appropriate set of states: the subspace of physical states and show that, indeed, a minimal uncertainty on the position occurs for such modified commutations relations.

\section{A proposal of physical state space}

As emphasized by K. M. M. the analysis of the physical implications of the operators leading to modified commutation relations needs a careful definition of them. Let us recall that the Hilbert space we consider is the space of square integrable functions with respect to the measure $d p / f(p)$. On this space, following K. M. M. we start by defining in a first step a symmetric position operator $\hat{x}$ given in $p$-representation by if $f(p) \partial_{p} \phi(p)$ with as domain $\mathcal{D}(\hat{x})$ the set of differentiable functions $\phi$, vanishing at infinity, and such that $\int_{-\infty}^{+\infty} f(p)\left|\partial_{p} \phi\right|^{2} d p$ exists. The extension of this operator to a self-adjoint operator depends on the behavior of the functions $f(p)$ and $z(p)$ when $|p|$ goes to infinity. Two cases have to be considered.

\subsection{The compact case}

Let us suppose that $f(|p|)$ grows at infinity like $|p|^{1+\nu}$, with $\nu>0$, and therefore that $z(p)$ (eq. (8)) goes to finite limits when $|p| \rightarrow \infty$ :

$$
z(+\infty)=\alpha_{+}>0 \quad, \quad z(-\infty)=\alpha_{-}<0 \quad .
$$

In this case, the mapping $p \mapsto z(p)$ defines a diffeomorphism between $\mathbb{R}$ and the interval $] \alpha_{-}, \alpha_{+}\left[\right.$. The operator $\hat{x}$ is represented by $i \partial_{z}$ on this interval and acts on the space $\mathcal{L}^{2}\left(\left[\alpha_{-}, \alpha_{+}\right], d z\right)$ of square integrable functions vanishing at $\alpha_{-}$and $\alpha_{+}$and whose derivatives are also square integrable:

$$
\mathcal{D}(\hat{x})=\left\{\psi(z) \in \mathcal{L}^{2}\left(\left[\alpha_{-}, \alpha_{+}\right], d z\right) \quad \text { such that } \quad \psi\left(\alpha_{-}\right)=\psi\left(\alpha_{+}\right)=0\right\} .
$$

This operator admits a one-parameter family of self-adjoint extensions [9] $\hat{\mathbf{x}}_{\lambda}$ whose domains of definition now are the sets of $\mathcal{L}^{2}\left(\left[\alpha_{-}, \alpha_{+}\right], d z\right)$ functions with 
square integrable derivatives $\left\lceil\mathcal{W}^{1,2}\left(\left[\alpha_{-}, \alpha_{+}\right], d z\right)\right.$ such that:

$$
\psi\left(\alpha_{+}\right)=\exp (-i \lambda) \psi\left(\alpha_{-}\right) \quad .
$$

Each of these operators admits an infinite set of orthonormal eigenvectors:

$$
\begin{array}{lll}
\hat{\mathbf{x}}_{\lambda} \psi_{n}=\xi_{\lambda, n} \psi_{n} & \text { with } & \psi_{n}(z)=c \exp \left(-i \xi_{\lambda, n} z\right) \\
\xi_{\lambda, n}=\frac{\lambda+2 n \pi}{\alpha_{+}-\alpha_{-}} & \text {and } & |c|^{2}=\frac{1}{\alpha_{+}-\alpha_{-}}
\end{array}
$$

If $\nu>2$, these states not only have $\Delta \hat{\mathbf{x}}_{\lambda}=0$ but also present a finite uncertainty in momentum. Nevertheless there is no contradiction with the inequality (14). Indeed, while the state $\hat{\mathbf{A}}_{\ell} \psi_{n}$ is well defined, it did not belong to the domain of the operator $\hat{\mathbf{A}}_{\ell}^{\dagger}=\hat{\mathbf{A}}_{\ell}$; thus we may not infer the equality (14).

We also may use states represented in eq. (7). They are always normalizable when $\Lambda>0$ and for all values of $\Lambda$ if $u(+\infty)$ and $u(-\infty)$ are both finite. In this last case, the condition (2) is not verified, but even if $\lim _{p \rightarrow \infty} u(|p|)=\infty$ there are the mean values of the operators $f(\hat{\mathbf{p}})=f(p)$ and $\hat{\mathbf{x}}^{2}=\left(i f(p) \partial_{p}\right)^{2}$ that diverge and invalidate the derivation of the equalities (13 14). Contrariwise, if $u(+\infty)$ and $u(-\infty)$ diverge (for instance if $\nu \leq 1),(\Delta \hat{\mathbf{x}})^{2}$ will be finite and will possibly present a minimum. What we learn from the previous discussion is that maximally localized states have not necessarily to belong to the set of squeezed states, but must be, as least, in the intersection of the domains of the operators $\hat{\mathbf{x}}_{\lambda}$ and $\hat{\mathbf{x}}^{2}=-\partial_{z}^{2}$. Now comes into the game a new subtlety: the domain that makes this operator $\hat{\mathbf{x}}^{2}$ self-adjoint can also be defined in different ways. The most obvious one is to consider $\hat{\mathbf{x}}_{\lambda}^{2}$, whose domain is given by :

$$
\begin{aligned}
\mathcal{D}\left(\hat{\mathbf{x}}_{\lambda}^{2}\right)= & \mathcal{D}\left(\hat{\mathbf{x}}_{\lambda}\right) \cap \text { Image }\left(\hat{\mathbf{x}}_{\lambda}\right) \\
= & \left\{\Psi(z) \in \mathcal{W}^{2,2}\left(\left[\alpha_{-}, \alpha_{+}\right], d z\right) \text { such that } \Psi\left(\alpha_{-}\right)=\exp [i \lambda] \Psi\left(\alpha_{+}\right)\right. \\
& \text {and } \left.\Psi^{\prime}\left(\alpha_{-}\right)=\exp [i \lambda] \Psi^{\prime}\left(\alpha_{+}\right)\right\}
\end{aligned}
$$

Let us tentatively define maximally localized states as those minimizing $\Delta \hat{\mathbf{x}}^{2}$ on $\mathcal{D}\left(\hat{\mathbf{x}}_{\lambda}^{2}\right)$ :

$$
\left(\Delta \hat{\mathbf{x}}^{\min }\right)^{2}=\min \frac{\left\langle\Psi\left|\hat{\mathbf{x}}_{\lambda}^{2}-\xi^{2}\right| \Psi\right\rangle}{\langle\Psi \mid \Psi\rangle} \equiv \mu^{2} \quad, \quad \Psi \in \mathcal{D}\left(\hat{\mathbf{x}}_{\lambda}^{2}\right)
$$

with

$$
\xi=\frac{\left\langle\Psi\left|\hat{\mathbf{x}}_{\lambda}\right| \Psi\right\rangle}{\langle\Psi \mid \Psi\rangle}
$$

In $p$-representation this implies that the wave function $\Psi(p)$ has to obey the equation:

$$
\left[-\left[f(p) \partial_{p}\right]^{2}-\xi^{2}+2 a\left(i f(p) \partial_{p}-\xi\right)-\mu^{2}\right] \Psi(p)=0
$$

\footnotetext{
${ }^{5} \mathrm{By} \mathcal{W}^{n, 2}\left(\left[\alpha_{-}, \alpha_{+}\right], d z\right)$ are denoted the spaces, known as Sobolev spaces, of square integrable functions whose derivative are, up to order $n$, also square integrable and whose norms are $\|f\|^{2}=\int_{\alpha_{-}}^{\alpha_{+}}\left(|f(z)|^{2}+\sum_{\ell=1}^{n}\left|f^{\ell /}(z)\right|^{2}\right) d z$.
} 
where $a$ is a multiplicator introduced in order to take into account the mean position condition imposed on the state. The parameter $\mu^{2}$, which corresponds to the value of the extrema of the functional (32), can also be interpreted as a multiplicator taking into account the normalization constraint imposed on the states. The general solution of eq. (30), satisfying the boundary conditions (27) reads as:

$$
\Psi(p)=C \exp \left[i\left(\frac{\lambda+2 n \pi}{\alpha_{+}-\alpha_{-}}\right) z(p)\right] \quad, \quad n \in \mathbb{Z} \quad .
$$

These solutions are simultaneous eigenfunctions of the operators $\hat{\mathbf{x}}_{\lambda}$ and $\hat{\mathbf{x}}_{\lambda}^{2}$. Thus, in general, they do not satisfy the localization condition (29), unless $\xi$ is in the spectrum of $\hat{\mathbf{x}}_{\lambda}$, in which case $\mu^{2}$ is zero and there is no spread in position. The only way we see to cure this defect is to modify the domain of the operator $\hat{\mathbf{x}}^{2}$. Indeed other boundary conditions than (27) can be chosen, that also makes $-\partial_{z}^{2}$ a self-adjoint operator. We shall define the squared position operator as the self adjoint operator $[9] \hat{x}^{\dagger} \hat{x}$ whose domain is $\mathcal{D}(\hat{x})$ defined in eq. (22). The advantage of this choice is that the states so selected may naturally belong to the domain of some unbounded operator. More physically, following K. M. M., we shall impose on the set of states which we consider as physically relevant that they satisfy an extra condition, for example that they be of bounded energy. To achieve this requirement we consider an operator $v(\hat{\mathbf{p}})$ such that, in $p$-representation, the representing function $v(p)$ diverges and is not integrable. We define the physical states as those whose expectation value of this operator is finite and reformulate the variational principle as

$$
\begin{aligned}
& \left(\Delta \hat{\mathbf{x}}^{\text {min }}\right)^{2}=\min \frac{\left\langle\Psi\left|\hat{\mathbf{x}}^{2}-\xi^{2}\right| \Psi\right\rangle}{\langle\Psi \mid \Psi\rangle} \equiv \mu^{2} \quad \\
& \text { with } \quad \xi=\frac{\left\langle\Psi\left|\hat{\mathbf{x}}_{\lambda}\right| \Psi\right\rangle}{\langle\Psi \mid \Psi\rangle} \quad, \quad \gamma=\frac{\langle\Psi|v(\hat{\mathbf{p}})| \Psi\rangle}{\langle\Psi \mid \Psi\rangle} \quad, \quad \Psi \in \mathcal{D}(\hat{x}) .
\end{aligned}
$$

The Euler-Lagrange equation governing this new variational principle needs the use of one more Lagrange multiplicator and reads as:

$$
\left[-\left[f(p) \partial_{p}\right]^{2}-\xi^{2}+2 a\left(i f(p) \partial_{p}-\xi\right)+2 b(v(p)-\gamma)-\mu^{2}\right] \Psi(p)=0
$$

where $V(z(p))=v(p)$ is the $z$-representation of $v(\hat{\mathbf{p}})$. Of course it is impossible, for an arbitrary function $v(p)$, to write any exact solution for this equation excepted when $b=0$. In this case, due to the new boundary conditions, that have to be taken in order that the mean value of $V(z)$ exists, the solution we obtain is:

$$
\begin{aligned}
& \Psi(p)=C \exp [-i \xi z(p)] \sin \left\{\mu\left[z(p)-\alpha_{-}\right]\right\} \\
& |C|=\sqrt{\frac{2}{\alpha_{+}-\alpha_{-}}}, \quad \mu=n \frac{\pi}{\alpha_{+}-\alpha_{-}} \quad, \quad n \in \mathbb{N}_{0} .
\end{aligned}
$$

What is new with this solution is that it belongs to $\mathcal{D}(\hat{x})$ and thus is no longer an eigenstate of $\hat{\mathbf{x}}_{\lambda}$ (but, of course, belongs also to $\mathcal{D}\left(\hat{\mathbf{x}}_{\lambda}\right)$ ). Accordingly, the 
corresponding spread in position is non-vanishing but is given by

$$
\left.\Delta \hat{\mathbf{x}}^{\text {min }}\right|_{b=0}=\frac{\pi}{\alpha_{+}-\alpha_{-}} .
$$

But what we have obtained here is the minimal spread under the extra condition that

$$
\frac{\langle\Psi|V(\hat{\mathbf{p}})| \Psi\rangle}{\langle\Psi \mid \Psi\rangle}=\frac{2}{\alpha_{+}-\alpha_{-}} \int_{\alpha_{-}}^{\alpha_{+}} V(z) \sin ^{2}\left[\frac{\pi\left(z-\alpha_{-}\right)}{\alpha_{+}-\alpha_{-}}\right] d z \equiv \gamma_{0}
$$

For any other value of $\gamma$, we have, in principle, to discuss the general solution of eq. (34), a task that is virtually impossible. However, the fact that we obtain a universal solution, "whatever" is the function $v(p)$ may be the signal that something particular happens when $b=0$. Indeed, if we vary $\gamma$ by a small amount $\delta \gamma$, a second order perturbation calculation [10] leads to

$$
\begin{aligned}
& \left(\Delta \hat{\mathbf{x}}^{m i n}\right)^{2}=\left(\left.\Delta \hat{\mathbf{x}}^{m i n}\right|_{b=0}\right)^{2}+\frac{1}{4} \frac{\delta \gamma^{2}}{T^{2}}, \quad b=-\frac{1}{2} \frac{\delta \gamma}{T^{2}} \\
& \text { where } \quad T^{2}=\frac{2\left(\alpha_{+}-\alpha_{-}\right)^{2}}{\pi^{2}} \sum_{n=2}^{\infty} \frac{V_{0 n}^{2}}{n^{2}-1} \\
& \text { and } \quad V_{0 n}=\int_{\alpha_{-}}^{\alpha_{+}} V(z) \sin \left[\frac{\pi\left(z-\alpha_{-}\right)}{\alpha_{+}-\alpha_{-}}\right] \sin \left[n \frac{\pi\left(z-\alpha_{-}\right)}{\alpha_{+}-\alpha_{-}}\right] d z
\end{aligned}
$$

indicating that $\left.\Delta \hat{\mathbf{x}}^{\text {min }}\right|_{b=0}$ corresponds to a (local) minimum with respect to $\gamma$. Such a formal calculation of course is just indicative, but it allows to conjecture that if we restrict ourselves to the class of physical states defined as those belonging to the domain $\mathcal{D}(\hat{x})$ we obtain a minimal length having a physical meaning. We may also understand this result in a geometrical way. We have more freedom when we search for the minimum of $\mu^{2}$ in $\mathcal{D}(\hat{x})$, without imposing the value of $\gamma$ than we have for a prescribed value of it. Therefore the minimum so obtained must be less or equal to those corresponding to a specified value of $\gamma$. Note also that as we have assumed a priori that the solution, belonging to $\mathcal{D}(\hat{x})$, obtained when $b=0$ defines a finite expectation value of the operator $v(\hat{\mathbf{p}})$, this implies that the function $v(p)$, defining the physical space, cannot diverge faster than $|p|^{3 \nu}$. If this condition is not satisfied we have to solve eq. (34) for arbitrary values of $b$, determine the set of value of $b$ that lead to finite expectation values of $v(\hat{\mathbf{p}})$ and then minimize the values of $\Delta \hat{\mathbf{x}}_{b}$ with respect to $b$.

\subsection{The non-compact case}

From the discussion of the previous subsection it is clear that if $\alpha_{-}$or $\alpha_{+}$become infinite, i.e. if $\nu<0$ the squeezed states define, for $\Lambda>0$, physical states (with respect to any polynomial operator $v(\hat{\mathbf{p}}))$ whose spread in position can be made as small as we want. Therefore there is no minimal length in this case. The semi bounded case (corresponding to one of the $\alpha$ finite and the other not) will not be considered here. 


\section{Two physical illustrations}

The previous analysis may appear to be formal and far from our physical apprehension of localization in $(x)$-space. So, to convince the reader that our approach is well founded, let us briefly discuss the quantization of a particle in a box [11] and the asymptotic behavior of the energy eigenvalues of the harmonic oscillator [2].

Of course, we only have to consider the case that we have called compact, the one where the function $z(p)$ tends to finite value when $|p|$ goes to infinity. Let us introduce the inverse of this function, a function $\zeta(z)$ such that

$$
\zeta[z(p)] \equiv p
$$

Note that this function becomes singular in $\alpha_{-}$and $\alpha_{+}$. In $x$-space we may represent (formally) the $\hat{\mathbf{x}}$ and $\hat{\mathbf{p}}$ operators as:

$$
\hat{\mathbf{x}}=x \quad, \quad \hat{\mathbf{p}}=\zeta\left(-i \partial_{x}\right) \quad .
$$

For the problem of quantizing a particle of mass $m$ in a box of length $L$, we may take as domain the square integrable, $C^{\infty}, \mathcal{L}^{2}([0, L], d x)$ functions, vanishing at $x=0$ and $x=L$. To simplify the discussion, let us first assume that the function $f(p)$ is even and therefore that $\zeta$ is odd. We have to solve the eigenvalue equation:

$$
\hat{\mathbf{p}}^{2} \Psi=2 m E \Psi \quad \Leftrightarrow \quad \zeta\left(-i \partial_{x}\right)^{2} \psi(x)=2 m E \psi(x)
$$

whose solutions are

$$
\psi(x) \propto \sin (k x) \quad \text { with } \quad k L=n \pi, n \in \mathbb{N}_{0} \quad \text { and } \quad 2 m E=\zeta(k)^{2}
$$

Imposing that the energy is bounded and is a monotone function of the number of nodes of the wave function, we deduce the existence of an upper bound $N_{\max }$ on the number of nodes and therefore of the dimensionality of the Hilbert space of eigenstates:

$$
N_{\text {max }}=\left[\alpha_{+} \frac{L}{\pi}\right]
$$

where, as usual, the brackets represent the function "integer part". This illustrates the existence of a minimal length in the theory: distances less than $\pi / \alpha_{+}$ are not distinguishable. If the function $\zeta$ is not odd, the eigenvalue problem (43) is solved as follows. For definiteness, let us assume $\alpha_{+}<\alpha_{-}$. We divide the interval $\left[0, \alpha_{+}\right]$into subintervals of length $\pi / L$. In each of such subinterval we may find a value of $k_{+}$such that there exists a value of $k_{-}$obeying the condition

$$
k_{-}=-k_{+} 2 n \frac{\pi}{L} \quad n \in \mathbb{Z} \quad \text { so that } \quad \zeta\left(k_{+}\right)=-\zeta\left(-k_{-}\right) \quad .
$$

The solutions of eq. (43) are therefore of the form:

$$
\psi(x) \propto \exp \left(i k_{+} x\right)-\exp \left(-i k_{-} x\right)
$$


with $k_{+} L \in[(n-1) \pi, n \pi], 1 \leq n<\alpha_{+} \frac{\pi}{L} \quad$ and $2 m E=\zeta\left(k_{+}\right)^{2}=\zeta\left(k_{-}\right)^{2}$.

The monotonic behavior of the function $\zeta(d \zeta(z) / d z=f[\zeta(z)])$ insures the existence and uniqueness of these solutions for each value of $n$.

We also may easily obtain the asymptotic behavior of the energy eigenvalues of an harmonic oscillator. The eigenvalue problem to be solved is

$$
\left(\frac{\hat{\mathbf{p}}^{2}}{2 m}+\frac{m \omega^{2}}{2} \hat{\mathbf{x}}^{2}\right) \Psi_{n}=E_{n} \Psi_{n}
$$

In term of the $z$ variable this problem is formally equivalent to the usual problem of a particle of mass $1 / m \omega^{2}$, in a potential that diverges like $\left|\alpha_{ \pm}-z\right|^{\nu_{ \pm}}$. For large value of the energy $E_{n}$ we may approximate this as the motion of a free particle in a box of size $\alpha_{+}-\alpha_{-}$. Accordingly, the asymptotic eigenvalues of the energy are given by:

$$
E_{n} \approx \frac{m \omega^{2}}{2}\left(\frac{\pi}{\alpha_{+}-\alpha_{-}} n\right)^{2}
$$

in accordance with the special case explicitly integrated in ref. [2]. The physical interpretation of this result [12] is the following. When the wave function, confined in an $x^{2}$ potential, presents a large number $n$ of nodes its spatial extension is of the order on $n\left|\Delta \hat{\mathbf{x}}^{\min }\right|=n \frac{\pi}{\alpha_{+}-\alpha_{-}}$and its energy is, for large values of $n$, approximated by

$$
E_{n} \approx \frac{m \omega^{2}}{2}\left(n\left|\Delta \hat{\mathbf{x}}^{m i n}\right|\right)^{2}
$$

contrary to the standard case where $\Delta \hat{\mathbf{x}}=\sqrt{\hat{\mathbf{x}}^{2}} \approx n / \Delta \hat{\mathbf{p}} \mid$ and $\Delta \hat{\mathbf{p}}^{2}=\hat{\mathbf{p}}^{2} \approx$ $m E_{n}$, leading to $E_{n} \approx n \omega$ for large value of $n$.

These examples already appear in the works [11, 2], assuming $f(p)=1+p^{2}$. In this case, as we have emphasized in this article, one may also find maximal localization using squeezed states. Our point here is that one may derive maximal localization from analogous studies of the wave equation with more general functions $f(p)$, only subject to the conditions (21), in which case these maximally localized states are not squeezed states, but they can be derived from the variational principle exhibited in (32, 33). Furthermore the above techniques applied to the asymptotic part of the spectrum of the wave equation is clearly generalizable to any potential function which is sufficiently slowly varying in $x$ space.

\section{Maximally localized states in $n$-dimension}

The way to extend the commutation relation (2) to $n$ dimensions is not unique. Let us now assume that $\hat{\mathbf{x}}$ and $\hat{\mathbf{p}}$ (resp. $\mathbf{x}$ and $\mathbf{p}$ ) are vector-valued operators 
(resp. vectors) of components $\hat{\mathbf{x}}_{j}$ and $\hat{\mathbf{p}}_{k}$ (resp. $\quad \mathbf{x}_{j}$ and $\mathbf{p}_{k}$ ) that obey the commutation relations

$$
\left[\hat{\mathbf{x}}_{j}, \hat{\mathbf{p}}_{k}\right]=i\left[f\left(\hat{\mathbf{p}}^{2}\right) \delta_{j k}+g\left(\hat{\mathbf{p}}^{2}\right) \hat{\mathbf{p}}_{j} \hat{\mathbf{p}}_{k}\right] \equiv i \hat{\Theta}_{j k},
$$

which ensure both translation and rotation invariance. Requiring the position operator components to satisfy $\left[\hat{\mathbf{x}}_{j}, \hat{\mathbf{x}}_{k}\right]=0$ further imposes the constraint (see [13]

$$
g=\frac{2 f \partial_{p^{2}} f}{f-2 p^{2} \partial_{p^{2}} f} .
$$

Similarly to what has been done in one dimension where $z(p)$ acted on the space $\mathcal{L}^{2}\left(\left[\alpha_{-}, \alpha_{+}\right], d z\right)$ as an operator such that $[\hat{\mathbf{x}}, \hat{\mathbf{z}}]=i$, we introduce the operator $\hat{\mathbf{z}}$ whose components are canonically conjugated to the components of the position operator :

$$
\left[\hat{\mathbf{x}}_{j}, \hat{\mathbf{z}}_{k}\right]=i \delta_{j k}
$$

represented by functions $\mathbf{z}_{j}(\mathbf{p})$ in $p$-representation. We can therefore, by representing $\hat{\mathbf{p}}_{j}$ as the functions $\zeta_{j}(\mathbf{z})$ in (51), rewrite this commutation relation as

$$
\partial_{z_{j}} \mathbf{p}_{k}=i \Theta_{j k}
$$

Thus $\partial_{z_{j}} \mathbf{p}_{k}=-\partial_{z_{k}} \mathbf{p}_{j}$ and therefore there exists a scalar function $\mathcal{U}$ generalizing $[u(p)]$ (8) such that

$$
p_{j}=\partial_{z_{j}} \mathcal{U}
$$

We shall assume this function $\mathcal{U}$ to depend only on the variable $\mathbf{z}^{2} / 2$ in order to preserve rotation invariance. Denoting the derivative with respect to $\mathbf{z}^{2} / 2$ as

$$
\frac{\partial \mathcal{U}}{\partial\left(\mathbf{z}^{2} / 2\right)}=\mathcal{U}^{\prime}
$$

we deduce from $(54)$ and $(55)$ that

$$
f(\mathbf{p})=\mathcal{U}^{\prime}[\mathbf{z}(\mathbf{p})] \quad \text { and } \quad g(\mathbf{p})=\frac{\mathcal{U}^{\prime \prime}[\mathbf{z}(\mathbf{p})]}{\mathcal{U}^{\prime}[\mathbf{z}(\mathbf{p})]} \quad .
$$

Under the assumption that relation (55) can be inverted, we can, in principle, express $|\mathbf{z}|$ as a function of $|\mathbf{p}|:|\mathbf{z}|=\mathcal{Z}(|\mathbf{p}|)$ and we may check that equation (52) is indeed verified. Furthermore eq. (55) defines a diffeomorphism between $\mathbb{R}^{n}$ and the $n$-dimensional ball :

$$
\mathcal{B}^{n}=\left\{\mathbf{z} \in \mathbb{R}^{n} \quad \text { such as } \quad|\mathbf{z}|<\rho_{+}\right\}
$$

where $\mathcal{Z}(+\infty)=\rho_{+}<+\infty$ in the compact case, the only one we consider here after.

A useful representation of the algebra displayed in (51) and (53), is on the Hilbert space $\mathcal{L}^{2}\left(\mathcal{B}^{n}, d^{n} z\right)$, as:

$$
\begin{aligned}
\hat{\mathbf{x}}_{j} \psi(\mathbf{z}) & =i \partial_{z_{j}} \psi(\mathbf{z}) \\
\hat{\mathbf{z}}_{j} \psi(\mathbf{z}) & =z_{j} \psi(\mathbf{z}) \\
\hat{\mathbf{p}}_{j} \psi(\mathbf{z}) & =z_{j} \mathcal{U}^{\prime}\left(\frac{\mathbf{z}^{2}}{2}\right) \psi(\mathbf{z})
\end{aligned}
$$


with $\psi(\mathbf{z})=\langle\mathbf{z} \mid \Psi\rangle$. K. M. M. suggested to build maximally localized states by considering the solution of the $n$ equations

$$
\hat{\mathbf{A}}_{j \Lambda}\left|\Psi_{\Lambda}\right\rangle \equiv\left[\left(\hat{\mathbf{x}}_{j}-\left\langle\hat{\mathbf{x}}_{j}\right\rangle\right)+i \Lambda\left(\hat{\mathbf{p}}_{j}-\left\langle\hat{\mathbf{p}}_{j}\right\rangle\right)\right)\left|\Psi_{\Lambda}\right\rangle=0
$$

where $\Lambda$ has to be independent of the index $j$ for the equations to be consistent. Their common solution reads as

$$
\psi(\mathbf{z}) \propto \exp \left[-i\langle\hat{\mathbf{x}}\rangle \mathbf{z}+\Lambda\langle\hat{\mathbf{p}}\rangle \mathbf{z}-\Lambda \mathcal{U}\left(\mathbf{z}^{2} / 2\right)\right] .
$$

But as we already discussed in the one-dimensional framework, this construction, excepted in a few special cases, does not lead to states with maximal localization or can even not be performed when no restriction ensuring that the operators $\hat{\mathbf{A}}_{i}$ and $\hat{\mathbf{A}}_{j}$ commute for all $i$ and $j$ is imposed on the commutation relations. In that case, we may decide to improve localization in some direction at the cost to deteriorate localization in another direction. As we illustrate below for the particular case $g\left(\hat{\mathbf{p}}^{2}\right)=1$ considered in [6] where the squeezed states construction can be achieved, those states do not display the minimal length uncertainty. Here again, our purpose will be to define maximally localized states as those minimizing $(\Delta \hat{\mathbf{x}})^{2}$ with $\langle\hat{\mathbf{x}}\rangle=\xi$ fixed and verifying suitable boundary conditions in order to be physically acceptable. Therefore, following the same procedure as in sect. (3), we find that the wave function reads as

$$
\psi(\mathbf{z})=\exp [-i \xi \cdot \mathbf{z}] \phi(\mathbf{z}) \quad \text { where } \quad\left(\boldsymbol{\Delta}+\mu^{2}\right) \phi(\mathbf{z})=0, \phi(\mathbf{z})_{\mid \partial \mathcal{B}^{n}}=0,
$$

$\xi$ denoting now the vector mean position and $\Delta$ the $n$-dimensional Laplacian operator on $\mathcal{B}^{n}$. The boundary condition $\psi(\mathbf{z})_{\left(|\mathbf{z}|=\rho_{+}\right)}=0$ leads to the quantization of $\mu^{2}$, whose smallest value is obtained for the $s$-wave. The corresponding wave function, bounded at the origin, is

$$
\psi(\mathbf{z})=C_{n} \exp [-i \xi . \mathbf{z}]|\mathbf{z}|^{-\nu} J_{\nu}(\mu|\mathbf{z}|) \quad \text { where } \quad \nu=\frac{n}{2}-1
$$

displaying the finite minimal uncertainty in position

$$
\Delta \hat{\mathbf{x}}^{\text {min }}=\frac{j_{\nu, 1}}{\rho_{+}} \quad,
$$

$j_{\nu, 1}$ being the first zero of the $J_{\nu}(x)$ Bessel function.

We end up this section by comparing, for illustrative purpose, the spread in position of the squeezed states obtained by K. M. M. in the particular case for which

$$
\mathcal{U}^{\prime}\left(\frac{\mathbf{z}^{2}}{2}\right)=\frac{1}{1-\mathbf{z}^{2} / 2}
$$

with the spread (64). The normalized squeezed states with $\langle\hat{\mathbf{x}}\rangle=\mathbf{0}$ and $\langle\hat{\mathbf{p}}\rangle=\mathbf{0}$ read as

$$
\psi_{\Lambda}(\mathbf{z})=\left[(2 \pi)^{-n / 2} \frac{\Gamma\left(2 \Lambda+\frac{n}{2}+1\right)}{\Gamma(2 \Lambda+1)}\right]^{1 / 2}\left(1-\frac{\mathbf{z}^{2}}{2}\right)^{\Lambda} .
$$


The squared uncertainty in position computed from these states, as a function of $\Lambda$, is

$$
\left(\Delta \mathbf{x}^{2}\right)=\frac{n \Lambda}{4} \frac{n+4 \Lambda}{2 \Lambda-1}
$$

whose minimum is

$$
\left(\Delta \hat{\mathbf{x}}_{\text {squeezed }}^{\text {min }}\right)^{2}=\frac{n}{8}\left(1+\sqrt{1+\frac{n}{2}}\right)\left(\frac{n+2}{\sqrt{1+\frac{n}{2}}}+2\right) \text { for } \Lambda=\frac{1}{2}+\frac{1}{2} \sqrt{1+\frac{n}{2}}
$$

is always greater than the one given by eq. (64). Incidentally, let us notice that from eqs (64) and (68) we obtain the inequality

$$
j_{\nu, 1}<\sqrt{\left(\nu+\frac{1}{2}\right)\left(\nu+2 \sqrt{\nu+\frac{3}{2}}+\frac{5}{2}\right)}
$$

which provides an upper bound to the first non-trivial zero of $J_{\nu}(x)$ when $2 \nu+1$ is an integer.

\section{Conclusions}

We have shown the emergence of a minimal length in quantum mechanics starting from very general assumptions on the commutation relations. We have clarified the meaning of maximally localized states, using to build them the most natural definition we may consider: a variational principle minimizing the spread of the state in space. We have also shown that the K. M. M. construction of such states in terms of squeezed states in general fails to provide maximally localized states. A particularly interesting physical consequence of our discussion is that in the regime where the minimal length becomes sensitive, the physics seems to become independent of the details of the modification of the commutation relations and depends only on the convergence of the integral $\int_{-\infty}^{+\infty} f(p)^{-1} d p$, as our analysis of the free particle and the harmonic oscillator examplifies. Our definition of maximally localized states extends also, without difficulties, to any number of dimensions. As a mathematical by-product of our discussion, we obtain a large class of majoration of the first zero of a family of Bessel functions. Indeed for each choice of the function $\mathcal{U}$ we may obtain a relation like eq. (69), the one displayed in the text being better than the Schafheitlin's one (for the values of the index $\nu$ considered here) [14].

\section{Acknowledgments}

We thank A. Kempf for many valuable comments on a preliminary version of this paper and for having drawn our attention on one of its previous work in

which a similar variational principle was considered to determine the state of lowest momentum uncertainty for a particle in a box (see sect. 2.1 of ref. 115]). 
We are also particular grateful to M. Lubo for stimulating discussions and comments. Moreover, one of us (Ph.S.) would like to thanks its colleagues R. Brout, C. Demol and C. Troestler for enlightening conversations. Ph.S. acknowledges support from the Belgian Fonds National de la Recherche Scientifique (FRFC contracts).

\section{A Appendix: an a priori minoration of $\Delta \hat{\mathbf{x}}$}

If $f(\hat{\mathbf{p}})$ can be expressed as a series of even power of $\hat{\mathbf{p}}$, with positive coefficients:

$$
f(\hat{\mathbf{p}})=1+\sum_{n=1}^{\infty} c_{n} \hat{\mathbf{p}}^{2 n} \quad \text { with } \quad c_{n} \geq 0 \quad .
$$

we obtain

$$
\langle f(\hat{\mathbf{p}})\rangle \geq 1+\sum_{n=1}^{\infty} c_{n}\left\langle\hat{\mathbf{p}}^{2}\right\rangle^{n}
$$

The proof of this inequality leads on the positivity of

$$
\left\langle\left(\hat{\mathbf{p}}^{2}-\left\langle\hat{\mathbf{p}}^{2}\right\rangle\right)^{2} \hat{\mathbf{p}}^{2 n-4}\right\rangle \geq 0
$$

from which we deduce that

$$
\left\langle\hat{\mathbf{p}}^{2 n}\right\rangle \geq 2\left\langle\hat{\mathbf{p}}^{2}\right\rangle\left\langle\hat{\mathbf{p}}^{2 n-2}\right\rangle-\left\langle\hat{\mathbf{p}}^{2}\right\rangle^{2}\left\langle\hat{\mathbf{p}}^{2 n-4}\right\rangle
$$

and by recurrence that

$$
\left\langle\hat{\mathbf{p}}^{2 n}\right\rangle \geq\left\langle\hat{\mathbf{p}}^{2}\right\rangle^{n} \quad .
$$

As a consequence, when formula (18) is valid, we deduce a lower bound for the squared position uncertainty on squeezed states :

$$
(\Delta \hat{\mathbf{x}})^{2} \geq(\Delta \hat{\mathbf{x}})_{\min }^{2}=\frac{1}{4} \min \frac{f^{2}\left(\sqrt{\left\langle\hat{\mathbf{p}}^{2}\right\rangle}\right)}{\left\langle\hat{\mathbf{p}}^{2}\right\rangle} .
$$

\section{References}

[1] A. Kempf, J. Math. Phys. 35, 4483 (1994), hep-th/9311147

[2] A. Kempf, G. Mangano, R. Mann, Phys. Rev. D 52 (1995) 1108, hep$\operatorname{th} / 9412167$

[3] A. Kempf,J. Math. Phys. 38, 1347 (1997), hep-th/9602085

[4] P. K. Townsend, Phys. Rev. D15, 2795 (1976),D. Amati, M. Cialfaloni, G. Veneziano, Phys. Lett. B216, 41 (1989), F. Lizzi, N. Mavromatos, Phys.Rev. D 55 (1997) 7859, hep-th 9611040

[5] L. Garay, Int. J. Mod. Phys. A 10 (1995) 145 
[6] A. Kempf, G. Mangano, Phys. rev. D 55 (1997) 7909, hep-th/9612084

[7] A. Kempf, Phys. Rev. D 63 083514, astro-ph/0009209

[8] A. Kempf, J.C. Niemeyer, Phys.Rev. D 64 (2001) 103501, astro$\mathrm{ph} / 0103225$

[9] F. Riesz, B. Sz-Nagy, Leçons d'analyse fonctionnelle, Gauthier-Villars, Paris (1955) sect. 119

[10] L. Landau, E. Lifchitz, Quantum Mechanics, Pergamon Press, London (1959) chapt. 6

[11] R. Brout, Cl. Gabriel, M. Lubo, Ph. Spindel, Phys. Rev. D 59 (1999) 044005, hep-th/9807063

[12] R. Brout, private communication.

[13] A. Kempf, J.Phys. A30 (1997) 2093, hep-th/9604045

[14] G. N. Watson, Theory of Bessel Functions, Cambridge University Press (1966) chap. 15

[15] A. Kempf, Phys. Rev. D 63 (2001) 024017 